\title{
Effects of Stubborn Agents on Bounded Confidence Opinion Dynamic systems: Unanimity in Presence of Stubborn Agents
}

\author{
Koresh Khateri \\ PhD Student \\ Electrical Engineering \\ Shahid Beheshti Univ. \\ Tehran, Iran, \\ 1983963113 \\ Email: \\ k khateri@sbu.ac.ir
}

\author{
Mahdi Pourgholi \\ Assistant Professor \\ Electrical Engineering \\ Shahid Beheshti Univ. \\ Tehran, Iran, \\ 1983963113 \\ Email: \\ m pourgholi@sbu.ac.ir
}

\author{
Mohsen Montazeri \\ Assistant Professor \\ Electrical Engineering \\ Shahid Beheshti Univ. \\ Tehran, Iran, \\ 1983963113 \\ Email: \\ m montazeri@sbu.ac.ir
}

\author{
Lorenzo Sabattini \\ Associate Professor \\ ARS Control Lab. \\ UNIMORE Univ. \\ Reggio Emilia, Italy, \\ 42122 \\ Email: \\ lorenzo.sabattini@unimore.it
}

\begin{abstract}
In this paper, various bounded confidence opinion dynamic algorithms are examined to illustrate the effect of a stubborn minority groups on opinion dynamics. A notion of variable opinion stubborn agent is defined and it is shown that stubborn minorities are able to fully control the opinions of a Hegselmann-Krause opinion dynamic system through deliberate slow variation in the opinions of stubborn agents. Furthermore, an upper bound for the change rate of stubborn agents to preserve connectivity and control other flexible agents is given. Moreover, a method based on population and growing confidence bound is presented to achieve both unanimity and stubborn opinion rejection. To support the proposed method simulation results are provided.
\end{abstract}

Keywords- opinion dynamic; stubborn agent; multi-agent systems; bounded confidence; Hegselmann-Krause model.

\section{INTRODUCTION}

One of the prerequisites for agents to cooperate in a multiagent platform is to make several agreements through their missions. These agreements should be based on the opinion of the majority or a combination of all opinions. However, an external agent or a stubborn agent in the group is able to severely affect the agreement process and a minority of stubborn agents are able to take opinion control from a majority of flexible agents. Therefore, their underlying opinion dynamic should be robust against such effects. An Example of a flexible majority with non-flexible minority can be found in food industry such as Halal or Kosher foods which are taking more proportion in the food groceries than the proportion of religious people in a given area with non-religious majorities [1]. Therefore, the nonflexible minority could have a substantial role in the decision-making process and such a condition is called the dictatorship of the small minority.

In the book, the wisdom of crowds [2], it is shown how averaging of guesses about an ox weight is extremely close to its actual value and introduces local averaging as a distributed method to make a consensus. However, an agent who is deciding on its opinion irrespective of other agents, can act as a leader. Such an agent is called stubborn agent hereafter in this paper. Authors in $[3,4]$ have illustrated how in a connected graph with local averaging agents, the final value of all agents' opinion will approach a convex combination of stubborn agents irrespective of their initial opinions. Therefore, the existence of stubborn agents in a group of averaging agents will destroy the benefits of an averaging system. In [5] a bounded confidence model is given by Hegselmann and Krause in which agents with opinions only in a bounded confidence can have effect on each other. Therefore, this approach will ignore stubborn agent intrusion, out of the confidence bound for each agent. Bounded confidence opinion dynamic model is a specific type of distance dependent networks [6] and connectivity preservation of these networks is investigated in [7, 8]. A modification on H-K (HegselmannKrause) model with a decaying confidence bound, in which only agents who follow another agent faster than a certain speed average their values, is given in [9]. In this scheme, agents will get more and more stubborn through the time. Thus, with this method a tradeoff exists between averaging benefits and stubborn agent isolation. Authors in [10] have proved a fix point asymptotic convergence of $\mathrm{H}-\mathrm{K}$ model in presence of any number of close minded agents. Moreover, a modification on $\mathrm{H}-$ $\mathrm{K}$ system is given in [11] such that connectivity of agents is preserved and unanimity is achieved. This modification considers the assumption that any agent initially is a neighbor at least to another agent and agents interact slowly enough to preserve their initial connectivity.

This paper shows how close minded agents could affect various opinion dynamic models and a method to avoid intolerance minority dictatorship is presented, an opinion is considered to be a bounded real-valued scalar and the stubborn agent effect on various bounded confidence opinion dynamic algorithms are examined.

The rest of the paper is organized as follows: Section II will inspect pure averaging, bounded confidence and decaying bounded confidence opinion dynamic systems in presence of stubborn agents. Section III presents our algorithm to achieve unanimity and isolation of stubborn agents, which we call population base growing confidence opinion dynamic. Finally, section IV concludes the paper. 


\section{EFFECT OF STUBBORN AGENTS ON OPINION DYNAMICS}

\section{A. pure averaging system}

Consensus in distributed pure averaging systems has been analyzed in $[12,13]$ and dynamic behavior model of each agent is represented in (1) where any flexible agent averages its own opinion with any other neighbors, irrespective of their opinion and only based on their adjacency relation, while stubborn agents arbitrarily determine their opinion. Here $p_{i}(t)$ is the opinion of agent $i$ after $t$ iterations. $F$ and $S$ are the set of flexible and stubborn agents, respectively. $N_{i}$ is the set of neighbors of agent $i$ and the function $|$.$| returns cardinality for$ a set and magnitude for a real or complex number. $u_{i}(t)$ is a deliberate opinion, which a stubborn agent can get in the next iteration.

$$
\left\{\begin{array}{cc}
p_{i}(t+1)=\frac{1}{\left|N_{i}\right|} \sum_{j \in N_{i}} p_{j}(t) & i \in F \\
p_{i}(t+1)=u_{i}(t) & i \in S
\end{array}\right.
$$

Equation (2) Represents a discrete linear dynamic form of this dynamic system. Where $A, B, M, x$ and $u$ are state matrix, input matrix, the number of flexible agents, a vector stack of flexible agent's opinion and a vector stack of stubborn agents, respectively and $A_{i j}, B_{i j}$ are element $i j$ of matrices $A, B$.

$$
x(t+1)=A x(t)+B u(t)
$$

where

$$
\begin{aligned}
& A_{i j}=\left\{\begin{array}{cc}
\frac{1}{\left|N_{i}\right|} & j \in N_{i} \\
0 & \text { o.t }
\end{array}\right. \\
& B_{i j}=\left\{\begin{array}{cc}
\frac{1}{\left|N_{i}\right|} & j+M \in N_{i} \\
0 & \text { o.t }
\end{array}\right.
\end{aligned}
$$

Proposition 1: Assume all initial opinions to be finite, then flexible opinions will stay bounded and stubborn agents have the ability to arbitrarily determine the controllable part of flexible opinions.

Proof: $\quad \sum_{j} A_{i j}=1 \quad$ and $\quad A_{i i}=1 /\left|N_{i}\right|$, therefore $\sum_{j \neq i} A_{i j}=1-1 /\left|N_{i}\right|$. According to the Gershgorin circle theorem, $\lambda(A)$ (i.e. eigenvalues of matrix $A$ ) are in a circle with radius $1-1 /\left|N_{i}\right|$ and the circle's center is at $1 /\left|N_{i}\right|+0 j$ in the complex plane. Therefore, $|\lambda(A)| \leq 1$ and $A$ is always stable, which proofs boundedness of the system, given boundedness of initial opinions of all agents. It is well-known with linear system theory, that the controllable part of system (2) can be arbitrarily determined by $u(t)$ which means controllable states in vector $x(t)$ that are opinions of flexible agents are controllable by $u(t)$ which is opinion of stubborn agents.

According to proposition 1, only stubborn agents, irrespective of initial values of flexible agents, will determine steady state opinions. Figure 1 shows how a single stubborn agent is able to change any other opinion in a pure averaging system with a circular communication graph (i.e. each agent is in contact with exactly two other agents). The simulation is done over 10 agents with random initial opinions and a single stubborn agent with fix opinion.

\section{B. Hegselmann-Krause system}

One of the most popular mathematical models in opinion formation of multi-agent systems has been introduced by Hegselmann and Krause [5], that is an opinion dynamic model in which agents only interact with each other in a certain confidence bound. This model is developed to simulate fragmentation and polarization of opinions and effect of various type of agents in such a system has been analyzed. Authors in [14] have illustrated the effect of charismatic and radical agents, while [15] derived optimal controller for a leader and [16] has analyzed Bayesian decision makers in bounded confidence opinion dynamics. Also, various modifications are published to extend H-K model for various goals [11, 17-19].

Equation (3) represents a mathematical formulation of Hegselmann-Krause model, where $R_{i}$ is the confidence bound.

$$
\left\{\begin{array}{cc}
\sum_{p_{i}(t+1)=\frac{p_{j}(t)}{j \in\left|p_{i}-p_{j}\right|<R_{i}}} & \\
\left|\left\{j \in N|| p_{i}-p_{j} \mid<R_{i}\right\}\right| & \\
p_{i}(t+1)=u_{i}(t) & i \in S
\end{array}\right.
$$

Theorem 1: A variable cooperative stubborn group with a rate of change less than $M / N \min \left(R_{i}\right)$ is able to determine an arbitrary unanimous consensus of the whole group, with tolerance of $\pm \min \left(R_{i}\right)$ in finite time less than $\left[3 D N / 2 M \min \left(R_{i}\right)\right]+1 \quad$ iterations, where $D=\max (p)-\min (p)$ is the size of opinion space. $N, M$ are number of whole agents and number of stubborn agents, respectively. 


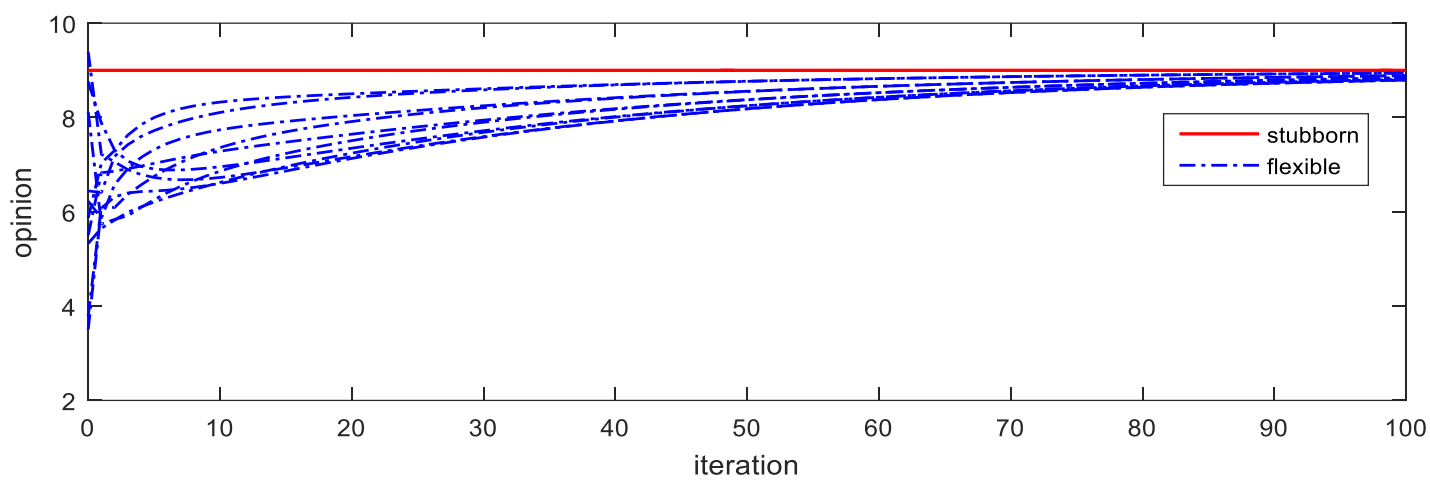

Figure 1 pure averaging system with a single stubborn agent

Proof: Consider all flexible agents to be in agreement initially with opinion $p_{f}$ and all stubborn agents to be cooperative and in agreement with opinion $p_{s}$, then:

$$
p_{f}(t+1)=\frac{N-M}{N} p_{f}(t)+\frac{M}{N} p_{s}(t)
$$

Flexible agents only interact with agents in their confidence bound. Therefore, $p_{s}(t)$ should stays in $\left[p_{f}(t)-\min \left(R_{i}\right), p_{f}(t)+\min \left(R_{i}\right)\right]$ interval to preserve connectivity with flexible agents, which makes sure all the agents to be interacting. Therefore, the best choice for stubborn agents is to choose one of the extreme points. Without losing generality, consider $p_{s}=p_{f}(t)+\min \left(R_{i}\right)$ then:

$$
\begin{gathered}
p_{f}(t+1)=\frac{N-M}{N} p_{f}(t)+\frac{M}{N}\left(p_{f}(t)+\min \left(R_{i}\right)\right) \\
p_{f}(t+1)-p_{f}(t)=\frac{M}{N} \min \left(R_{i}\right)
\end{gathered}
$$

Therefore:

$$
p_{s}(t+1)-p_{s}(t)=\frac{M}{N} \min \left(R_{i}\right)
$$

which means the maximum rate for stubborn agents, not to lose interaction with flexible agents, is less than $M / N \min \left(R_{i}\right)$. However, the case of initial unanimity of flexible agents is the worst case for stubborn agents. Otherwise, it was easier for stubborn agents to interact with a smaller group of flexible agents. Arbitrary unanimous consensus for stubborn agents to rule in $\mathrm{H}-\mathrm{K}$ system can be divided in two phases:

- Unanimity: in which stubborn agents collect flexible agents and determination, in which the group is guided toward an arbitrary point. Without losing generality consider final desired opinion is in the upper half of opinion space. In the first phase, stubborn agents should start at the minimum allowed opinion and increase their opinion with a rate less than $M / N \min \left(R_{i}\right)$ up to the maximum allowed opinion. This phase will be done at least in iterations less than $\left\lceil D N / M \min \left(R_{i}\right)\right\rceil$.

- Arbitrarily determination of the final opinion: After the unanimity phase, which in this case all agents will have the maximum allowed idea, stubborn agents will change their opinion to the desired final opinion with a rate less than $M / N \min \left(R_{i}\right)$, as we assumed that the desired opinion is in the upper half space of opinions, therefore it needs at most $\left[D N / 2 M \min \left(R_{i}\right)\right]$ iterations for the stubborn agent to arbitrarily determine the final opinion after the first phase.

Altogether, it needs a finite number of iterations less than $\left\lceil 3 D N / 2 M \min \left(R_{i}\right)\right\rceil+1$ for the $\mathrm{H}-\mathrm{K}$ system to be under unanimous consensus determined by stubborn agents. Figure 2 illustrates how a stubborn agent is able to make an arbitrary unanimous consensus in a bounded confidence system with random initial opinions in the interval [0-10] with uniform $R_{i}=1$ and the arbitrary opinion to be achieved is 8 .

\section{C. decaying bounded confidence system}

Authors in [9] has modified H-K model with a decaying bounded confidence. This modification leads to stubbornness of flexible agents throughout the time. A mathematical description of this modification is represented in (8).

$$
\left\{\begin{array}{c}
\sum_{i}(t+1)=\frac{j \in\left|p_{i}-p_{j}\right|<R_{i} e^{-c t}}{\left|\left\{j \in N|| p_{i}-p_{j} \mid<R_{i} e^{-c t}\right\}\right|} \\
p_{i}(t+1)=u_{i}(t)
\end{array} \quad i \in F\right.
$$




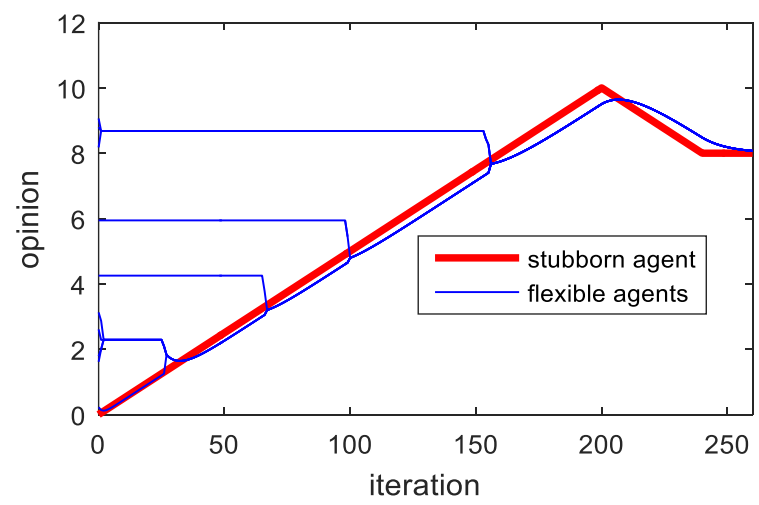

a)

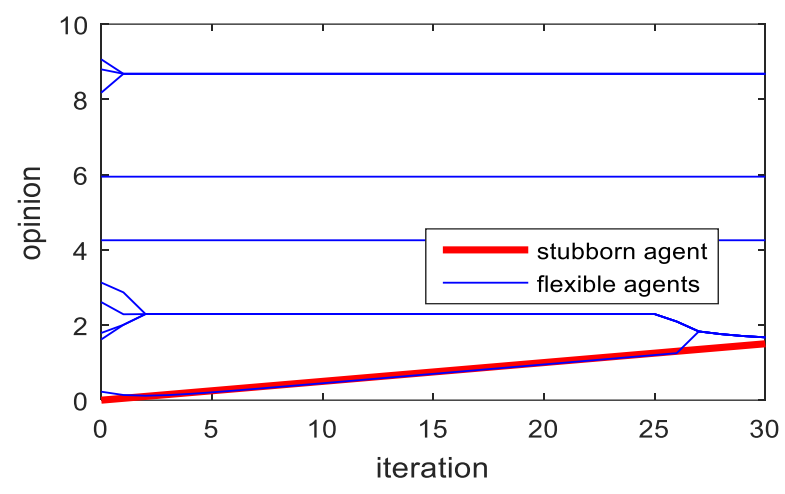

b)

Figure 2 a) stubborn rule out in $H$-K system b) the first 30 iterations magnified

Corollary1: A maximum rate less than $M / N^{\min }\left(R_{i} e^{-c t}\right)$ in each iteration is needed for stubborn agents to stay in contact with their flexible neighbors and there is no guarantee for a finite time arbitrary determination or unanimous consensus in this model.

Proof: Directly from theorem1 with the notion of exponential decaying of confidence bound.

Therefore, the rate of decaying confidence bound in this model provides a tradeoff between the maximum time of interaction and isolating stubborn agent effect. This approach will lead to more fragmentation of opinions and unanimous consensus will be far from reaching for a bigger decay rate of confidence bound. Also, a unique initial time for all agents is needed for this model and the system is going to be frozen as time elapse.

\section{POPULATION BASE GROWING CONFIDENCE BOUND METHOD}

This section provides a method to reject stubborn minorities, while achieving unanimity among flexible agents. It is known that confidence should be large to achieve unanimity in a bounded confidence dynamic system while large confidence is a gateway for stubborn agents to intrude and this tradeoff is intrinsic in such systems. To resolve this tradeoff, population of an opinion can be defined as the number of agents, which have that opinion in a given bound. If interaction protocol is such that only opinions, with more followers, impact agents with less popular opinions then minorities will be isolated. Our proposed method uses a growing confidence bound so that agents will have an opportunity to make a local agreement at the beginning iterations while they are safeguarded against stubborn agents by mean of a small confidence bound. However, while the confidence bound grows, population of local agreements can be higher than number of stubborn agents, which provides a safeguard by means of population. Therefore, a population based growing confidence bound can be used to have benefits of the pure averaging system together with the benefits of decaying bound opinion dynamic systems.

In (9) $P\left(p_{i}\right)$ is a function which returns number of agents with opinion $p_{i}$ in a certain bound $R_{0}$. Therefore agents with difference in opinion less than $R_{0}$ are belonging to the opinion $p_{i}$. Also, confidence bound for each agent $R_{i}(t)$ grows exponentially until it reaches the size of opinion space and $\lambda_{i}>1$ is the growing confidence rate for agent $i$.

$$
\left\{\begin{array}{c}
p_{i}(t+1)=\frac{j \in\left|p_{i}-p_{j}\right|<R_{i}(t) \cap j \in N \mid P\left(p_{i}\right) \leq P\left(p_{j}\right)}{\left|\left\{j \in N|| p_{i}-p_{j}\left|<R_{i}(t) \cap j \in N\right| P\left(p_{i}\right) \leq P\left(p_{j}\right)\right\}\right|} \\
p_{i}(t+1)=u_{i}(t)
\end{array} \quad i \in F\right.
$$

where:

$$
\begin{gathered}
P\left(p_{i}\right)=\left|\left\{j|| p_{i}-p_{j} \mid<R_{0}\right\}\right| \\
\text { And } \\
\left\{\begin{array}{cc}
R_{i}(t+1)=\lambda_{i} R_{i}(t) & R_{i}(t) \leq D \\
R_{i}(t+1)=R_{i}(t) & \text { o.t }
\end{array}\right.
\end{gathered}
$$

Lemma 1 [13]: The necessary and sufficient condition for a multi agent system to reach unanimous consensus is strong connectivity of its communication digraph.

Crollary1: For $\lambda_{i}>1$ all flexible agents in (9) will always converge to a unanimous consensus.

Proof: For $\lambda_{i}>1, R_{i}(t) \geq D$ will occur after some finite iterations, and at least one opinion group $i$ will have $P\left(p_{i}\right) \geq P\left(p_{j}\right)$ for any other $j$, therefore after a finite time all agents will average their values at least with the majority group or groups. Therefore, the communication digraph is strongly connected after a finite time. Therefore, according to lemma 1, agents will have a unanimous agreement. All agents will converge to the majority group or a convex combination of majority groups in situations where there appear various majority opinion groups with equal followers. 
Proposition 2: For opinion dynamic (9), after formation of a majority group, finite time needed for stubborn agents to arbitrarily set the consensus could be arbitrarily large.

Proof: Stubborn agents should stay in the bound $\left[p_{g}(t)-R_{0}, p_{g}(t)+R_{0}\right]$ to be in the same group with $p_{g}$, therefore to preserve their group the maximum rate of opinion change for stubborn agents is $M / N_{N} R_{0}$ with the same argument in theorem 1 . With $R_{0}=0$, the time to arbitrarily determine final consensus value, $\left[3 D N / 2 M R_{0}\right\rceil+1$ could be infinitely large.

Remark 1: In a practical noisy analogue communication between agents $R_{0}=0$ is not acceptable, because it is virtually impossible to have agents with the same opinion and all of the opinion groups will only contain a single agent. However, in digital communications $R_{0}=0$ is possible.

Figure 3 illustrates interaction of 10 flexible agents with opinion dynamic (9) and a single stubborn agent, it shows that flexible agents will be in unanimous agreement without fragmentation while in its counterpart $\mathrm{H}-\mathrm{K}$ model with a small confidence bound there will exist polarization and the stubborn agent were able to attract flexible agents in their confidence bound.

\section{CONCLUSION}

Averaging system's vulnerability to stubborn agent effect has been analyzed in this paper. It has been shown that pure averaging systems could be under full control of stubborn agents. Even though bounded confidence systems are known to have robustness against external opinions, we have shown a finite time arbitrary determination process for opinion control in bounded confidence systems. In addition, decaying bound opinion dynamic has been analyzed and it showed less susceptibility against stubbornness. However, a decaying bound will lead to stubbornness of all agents and averaging benefits will be lost. Therefore, a method based on population and growing bounds have been presented which rejects stubborn agent effect at the beginning iterations by a small initial confidence bound while after formation of a majority group or groups we showed that minority of stubborn agents are unable to arbitrarily determine final opinion of the flexible agents.

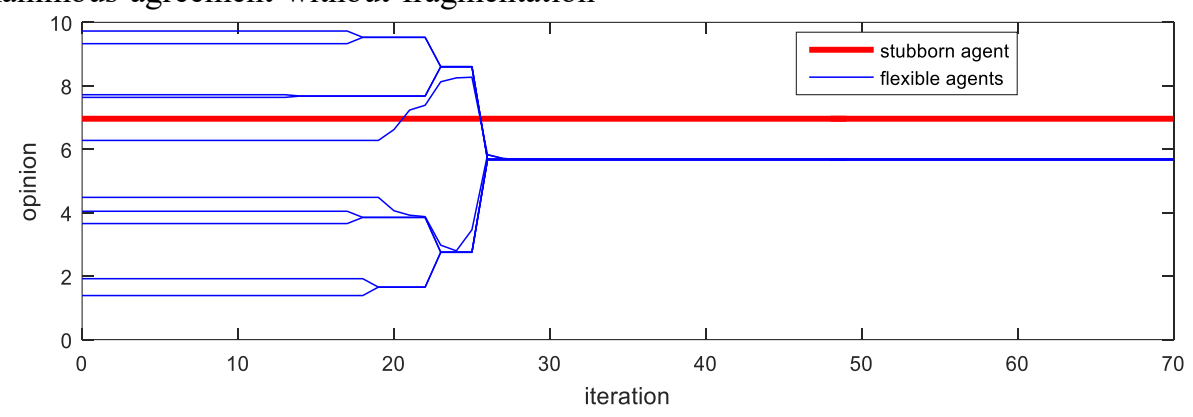

Figure 3 population base growing confidence opinion dynamic

\section{REFRENCES}

[1] N. Taleb, "The Most Intolerant Wins: The Dictatorship of the Small Minority," Incerto, 2016.

[2] J. Surowiecki, The wisdom of crowds. Anchor, 2005.

[3] J. Ghaderi and R. Srikant, "Opinion dynamics in social networks with stubborn agents: Equilibrium and convergence rate," Automatica, vol. 50, no. 12, pp. 3209-3215, 2014.

[4] R. Olfati-Saber and R. M. Murray, "Consensus problems in networks of agents with switching topology and time-delays," IEEE Transactions on automatic control, vol. 49, no. 9, pp. 15201533, 2004.

[5] R. Hegselmann and U. Krause, "Opinion dynamics and bounded confidence models, analysis, and simulation," Journal of artificial societies and social simulation, vol. 5, no. 3, 2002.

[6] G. Jing, Y. Zheng, and L. Wang, "Consensus of multiagent systems with distance-dependent communication networks," IEEE transactions on neural networks and learning systems, vol. 28, no. 11, pp. 2712-2726, 2017

K. Khateri, M. Pourgholi, M. Montazeri, and L. Sabattini, "A Comparison Between Decentralized Local and Global Methods for Connectivity Maintenance of Multi-Robot Networks," IEEE

Robotics and Automation Letters, vol. 4, no. 2, pp. 633-640, 2019.
L. Sabattini, C. Secchi, N. Chopra, and A. Gasparri, "Distributed control of multirobot systems with global connectivity maintenance," IEEE Transactions on Robotics, vol. 29, no. 5, pp. 1326-1332, 2013.
[9] I.-C. Mor and A. Girard, "Opinion dynamics with decaying confidence: Application to community detection in graphs," IEEE Transactions on Automatic Control, vol. 56, no. 8, pp. 1862-1873, 2011.

[10] B. Chazelle and C. Wang, "Inertial hegselmann-krause systems," IEEE Transactions on Automatic Control, vol. 62, no. 8, pp. 39053913, 2017.

[11] Y. Yang, D. V. Dimarogonas, and X. Hu, "Opinion consensus of modified Hegselmann-Krause models," Automatica, vol. 50, no. 2, pp. 622-627, 2014.

[12] T. Li and J.-F. Zhang, "Consensus conditions of multi-agent systems with time-varying topologies and stochastic communication noises," IEEE Transactions on Automatic Control, vol. 55, no. 9, pp. 2043-2057, 2010.

[13] R. Olfati-Saber, J. A. Fax, and R. M. Murray, "Consensus and cooperation in networked multi-agent systems," Proceedings of the IEEE, vol. 95, no. 1, pp. 215-233, 2007.

[14] R. Hegselmann and U. Krause, "Opinion dynamics under the influence of radical groups, charismatic leaders, and other constant signals: A simple unifying model," NHM, vol. 10, no. 3, pp. 477 $509,2015$.

[15] S. Wongkaew, M. Caponigro, and A. Borzi, "On the control through leadership of the Hegselmann-Krause opinion formation model," Mathematical Models and Methods in Applied Sciences, vol. 25, no. 03, pp. 565-585, 2015.

[16] K. R. Varshney, "Bounded confidence opinion dynamics in a social network of bayesian decision makers," IEEE Journal of 
Selected Topics in Signal Processing, vol. 8, no. 4, pp. 576-585,

2014.

[17] X. Chen, Z. Wu, H. Wang, and W. Li, "Impact of Heterogeneity on Opinion Dynamics: Heterogeneous Interaction Model," Complexity, vol. 2017, 2017.

[18] D. Mozyrska and M. Wyrwas, "Fractional discrete-time of Hegselmann-Krause's type consensus model with numerical simulations," Neurocomputing, vol. 216, pp. 381-392, 2016.

[19] T. Tao, Y. Zhao, and J. Hu, "On fuzzy Hegelsman-Krause opinion dynamics," in Control And Decision Conference (CCDC), 2017 29th Chinese, 2017, pp. 5290-5294: IEEE. 